\title{
Measurability of nonequilibrium thermodynamics in terms of the Hamiltonian of mean force
}

\author{
Philipp Strasberg $\oplus^{1}$ and Massimiliano Esposito $\circledast^{2}$ \\ ${ }^{1}$ Física Tè̀rica: Informació i Fenòmens Quàntics, Departament de Física, Universitat Autònoma de Barcelona, \\ 08193 Bellaterra (Barcelona), Spain \\ ${ }^{2}$ Complex Systems and Statistical Mechanics, Department of Physics and Materials Science, \\ University of Luxembourg, L-1511 Luxembourg, Luxembourg
}

(Received 24 January 2020; accepted 22 April 2020; published 11 May 2020)

\begin{abstract}
The nonequilibrium thermodynamics of an open (classical or quantum) system in strong contact with a single heat bath can be conveniently described in terms of the Hamiltonian of mean force. However, the conventional formulation is limited by the necessity to measure differences in equilibrium properties of the system-bath composite. We make use of the freedom involved in defining thermodynamic quantities, which leaves the thermodynamics unchanged, to show that the Hamiltonian of mean force can be inferred from measurements on the system alone, up to that irrelevant freedom. In doing so, we refute a key criticism expressed in the works by P. Talkner and P. Hänggi [Phys. Rev. E 94, 022143 (2016) and arXiv:1911.11660]. We also discuss the remaining part of the criticism.
\end{abstract}

DOI: 10.1103/PhysRevE.101.050101

\section{INTRODUCTORY REVIEW}

We start by reviewing recent progress in nonequilibrium strong-coupling thermodynamics based on the Hamiltonian of mean force [1-20] in a unified way. The goal is to find a consistent thermodynamic description for a system, which can be driven far away from equilibrium and which exchanges energy with a single arbitrary strongly coupled bath. If it can also exchange particles with the bath, similar constructions were independently proposed in Refs. [21-23]. We here focus only on the exchange of energy.

For this purpose we consider open systems specified by an arbitrary global Hamiltonian of the form $H_{S B}\left(\lambda_{t}\right)=H_{S}\left(\lambda_{t}\right)+$ $V_{S B}+H_{B}$. Here, $H_{S}\left(\lambda_{t}\right)$ is the system Hamiltonian, which can depend on an external driving protocol $\lambda_{t}$ (e.g., a changing electromagnetic field), $H_{B}$ is the bare bath Hamiltonian, and $V_{S B}$ describes the system-bath interaction. Note that a timedependent interaction $V_{S B}\left(\lambda_{t}\right)$ can be considered within the thermodynamic framework based on the Hamiltonian of mean force [11-14,18], but for ease of presentation we do not include this possibility here. In this Rapid Communication, we use a quantum-mechanical notation for convenience. If a result is only valid for classical systems, we explicitly emphasize it.

For the moment we consider the time $t$ to be fixed. If the global state of the system-bath composite is an equilibrium canonical state at inverse temperature $\beta=T^{-1}\left(k_{B} \equiv 1\right)$, it is given by $\pi_{S B}\left(\lambda_{t}\right) \equiv e^{-\beta H_{S B}\left(\lambda_{t}\right)} / \mathcal{Z}_{S B}\left(\lambda_{t}\right)$ with the partition function $\mathcal{Z}_{S B}\left(\lambda_{t}\right)=\operatorname{tr}\left\{e^{-\beta H_{S B}\left(\lambda_{t}\right)}\right\}$. In that case the corresponding reduced equilibrium state of the system is given by

$$
\pi_{S}^{*}\left(\lambda_{t}\right) \equiv \operatorname{tr}_{B}\left\{\pi_{S B}\left(\lambda_{t}\right)\right\}
$$

which is in general not of the canonical Gibbs form due to the non-negligible coupling $V_{S B}$, i.e., $\pi_{S}^{*}\left(\lambda_{t}\right) \neq e^{-\beta H_{S}\left(\lambda_{t}\right)} / \mathcal{Z}_{S}\left(\lambda_{t}\right)$. However, it can always be written in that canonical form with an effective Hamiltonian, which is known as the Hamil- tonian of mean force (HMF) [24,25] and which equals $-T \ln \pi_{S}^{*}\left(\lambda_{t}\right)$ up to an additive constant. A common and convenient choice for that constant is fixed using $H_{S}^{*}\left(\lambda_{t}\right)=$ $-T \ln \left[\mathcal{Z}_{S}^{*}\left(\lambda_{t}\right) \pi_{S}^{*}\left(\lambda_{t}\right)\right]$ with $[26,27]$

$$
\mathcal{Z}_{S}^{*}\left(\lambda_{t}\right) \equiv \frac{\mathcal{Z}_{S B}\left(\lambda_{t}\right)}{\mathcal{Z}_{B}} .
$$

Here, $\mathcal{Z}_{B} \equiv \operatorname{tr}_{B}\left\{e^{-\beta H_{B}}\right\}$ is the partition function of the bath alone. One should note that the $\operatorname{HMF} H_{S}^{*}\left(\lambda_{t}\right)=H_{S}^{*}\left(\lambda_{t}, \beta\right)$ depends on the inverse temperature.

In the following we recapitulate some essential elements of the nonequilibrium thermodynamics based on the HMF. For ease of presentation we assume that the initial system-bath state is described by the global equilibrium state $\pi_{S B}\left(\lambda_{0}\right)$, where we set the initial time to be $t=0$. Note that this initial state is different from the class of decorrelated initial states $\rho_{S B}(0)=\rho_{S}(0) \otimes \pi_{B}$, which is conventionally considered in the theory of open quantum systems [28] and requires a thermodynamic treatment not captured by the HMF [29-35]. The present framework is therefore particularly designed to treat initially correlated (and perhaps even entangled) system-bath states. Note that, classically, a larger class of correlated initial system states can be treated provided that the bath is initially in a conditional equilibrium state [6], but this result has no straightforward analog in the quantum regime [17]. However, if one pays attention to the fact that the state preparation itself has a thermodynamic cost, then any initial system state can be also treated within the HMF framework of strong-coupling thermodynamics [18]. Finally, a single framework combining both correlated and decorrelated initial states was proposed in Ref. [34].

Although we assume to start in equilibrium, we allow the driving protocol $\lambda_{t}$ to vary arbitrarily in time. This implies that the system-bath state at a later time $t>0$ is no longer in equilibrium, i.e., $\rho_{S B}(t) \neq \pi_{S B}\left(\lambda_{t}\right)$ in general. The mechanical 
work done on the system is identified as usual as

$$
W(t) \equiv \int_{0}^{t} d s \operatorname{tr}_{S}\left\{\frac{\partial H_{S}\left(\lambda_{s}\right)}{\partial s} \rho_{S}(s)\right\},
$$

where $\rho_{S}(t)=\operatorname{tr}_{B}\left\{\rho_{S B}(t)\right\}$ is the reduced state of the system at time $t$. The second law of nonequilibrium thermodynamics was found to be (for classical dynamics this was first derived in Ref. [6] and for quantum dynamics in Ref. [17])

$$
\Sigma(t) \equiv \beta\left[W(t)-\Delta F_{S}^{*}(t)\right] \geqslant 0
$$

with $\Delta F_{S}^{*}(t) \equiv F_{S}^{*}(t)-F_{S}^{*}(0)$. Here, the generalization of the nonequilibrium free energy to the strong-coupling regime is defined as

$$
F_{S}^{*}(t) \equiv \operatorname{tr}_{S}\left\{H_{S}^{*}\left(\lambda_{t}\right) \rho_{S}(t)\right\}+T \operatorname{tr}_{S}\left\{\rho_{S}(t) \ln \rho_{S}(t)\right\} .
$$

Furthermore, $\Sigma(t)$ is known as the entropy production and thus, Eq. (4) takes on the familiar form of phenomenological nonequilibrium thermodynamics [36] identifying the free energy (5) as the quantity, which gets minimized at equilibrium. The second law (4) can be also expressed in terms of the relative entropy $D[\rho \| \sigma] \equiv \operatorname{tr}\{\rho(\ln \rho-\ln \sigma)\}$ as [17]

$$
\Sigma(t)=D\left[\rho_{S B}(t) \| \pi_{S B}\left(\lambda_{t}\right)\right]-D\left[\rho_{S}(t) \| \pi_{S}^{*}\left(\lambda_{t}\right)\right],
$$

from which the non-negativity of $\Sigma(t)$ follows. Furthermore, if the dynamics are classical, it also holds that [17]

$$
\Sigma(t)=-\left.\int_{0}^{t} d s \frac{\partial}{\partial s}\right|_{\lambda_{s}} D\left[\rho_{S}(s) \| \pi_{S}^{*}\left(\lambda_{s}\right)\right] .
$$

Here, the derivative is taken with respect to a fixed $\lambda_{s}$. Finally, notice that the nonequilibrium free energy can be linked to the equilibrium free energy, denoted with a caligraphic letter $\mathcal{F}_{S}^{*}\left(\lambda_{t}\right)=-T \ln \mathcal{Z}_{S}^{*}\left(\lambda_{t}\right)$, via the relation

$$
F_{S}^{*}(t)-\mathcal{F}_{S}^{*}\left(\lambda_{t}\right)=T D\left[\rho_{S}(t) \| \pi_{S}^{*}\left(\lambda_{t}\right)\right] \geqslant 0 .
$$

Due to the non-negativity of relative entropy and since we assumed to start in equilibrium, Eq. (4) implies the weaker inequality

$$
W_{\text {diss }}(t) \equiv W(t)-\Delta \mathcal{F}_{S}^{*}\left(\lambda_{t}\right) \geqslant 0
$$

In this context $W_{\text {diss }}(t)$ is known as the "dissipated work" and Eq. (9) was first derived for classical dynamics in Ref. [1] and for quantum dynamics in Ref. [2].

Remarkably, Eq. (9) can be extended to a fluctuation theorem [1,2]

$$
\left\langle e^{-\beta w(t)}\right\rangle=e^{-\beta \Delta \mathcal{F}_{S}^{*}\left(\lambda_{t}\right)} .
$$

Classically, $\langle\cdots\rangle$ denotes an ensemble average over many trajectories and $w(t)$ is the stochastic work, which follows from evaluating Eq. (3) along a single trajectory (see Ref. [1] for details). Quantum mechanically, Eq. (10) can be derived using the so-called "two-point-projective-energy-measurement scheme" (TPPEMS; see Refs. [37,38] for reviews). Furthermore, for classical dynamics also Eq. (4) can be extended to a fluctuation theorem [6]:

$$
\left\langle e^{-\beta\left[w(t)-\Delta f_{S}^{*}(t)\right]}\right\rangle=1 .
$$

Here, $f_{S}^{*}(t)$ is the stochastic nonequilibrium free energy (see the Supplemental Material for more details and a proof of Eq. (11) [39]). The two fluctuation theorems (10) and (11) need to be distinguished in general. If the dynamics are such that for a fixed control parameter $\lambda_{t}$ the final nonequilibrium state $\rho_{S}(t)$ relaxes back to the equilibrium state (for instance, when the global system is weakly coupled to an ideal superbath), then Eq. (11) implies Eq. (10). Interestingly, a corresponding quantum version of Eq. (11) is not known to exist for general open system dynamics.

We now turn to the definition of internal energy, heat, and system entropy. We emphasize, however, that the second law (4), together with the definition of work, Eq. (3), is sufficient to characterize the set of allowed state transformations and the overall dissipation of the process. Indeed, it is clear from the basic definition of the nonequilibrium free energy,

$$
F_{S}^{*}(t)=\tilde{U}_{S}(t)-T \tilde{S}_{S}(t)
$$

that there are a priori many options to define an internal energy $\tilde{U}_{S}(t)$ (which fixes the definition of heat via the first law) or a thermodynamic entropy $\tilde{S}_{S}(t)$ of the system (which fixes the definition of heat via the second law), without having any impact on the second law. Furthermore, all that matters for the second law is the change in nonequilibrium free energy, which leaves us with a further freedom since Eq. (5) is only fixed up to an irrelevant constant value with respect to a standard reference state. We review two convenient choices.

One choice, which was used in Refs. [6,10,12,14,17,18,20] to construct a framework of nonequilibrium thermodynamics, identifies

$$
\begin{gathered}
\tilde{U}_{S}(t) \equiv U_{S}^{*}(t)=\operatorname{tr}_{S}\left\{\rho_{S}(t)\left[H_{S}^{*}\left(\lambda_{t}\right)+\beta \partial_{\beta} H_{S}^{*}\left(\lambda_{t}\right)\right]\right\}, \\
\tilde{S}_{S}(t) \equiv S_{S}^{*}(t)=\operatorname{tr}_{S}\left\{\rho_{S}(t)\left[-\ln \rho_{S}(t)+\beta^{2} \partial_{\beta} H_{S}^{*}\left(\lambda_{t}\right)\right]\right\},
\end{gathered}
$$

which requires one to evaluate the partial derivative $\partial_{\beta} H_{S}^{*}\left(\lambda_{t}\right)$. Furthermore, starting with $\mathcal{F}_{S}^{*}\left(\lambda_{t}\right)=-T \ln \mathcal{Z}_{S}^{*}\left(\lambda_{t}\right)$, a straightforward calculation reveals that [40]

$$
\mathcal{U}_{S}^{*}\left(\lambda_{t}\right)=\partial_{\beta}\left[\beta \mathcal{F}_{S}^{*}\left(\lambda_{t}\right)\right], \quad \mathcal{S}_{S}^{*}\left(\lambda_{t}\right)=\beta^{2} \partial_{\beta} \mathcal{F}_{S}^{*}\left(\lambda_{t}\right) .
$$

Here, $\mathcal{U}_{S}^{*}\left(\lambda_{t}\right)$ and $\mathcal{S}_{S}^{*}\left(\lambda_{t}\right)$ are the equilibrium counterparts of $U_{S}^{*}(t)$ and $S_{S}^{*}(t)$ obtained by replacing $\rho_{S}(t)$ with $\pi_{S}^{*}\left(\lambda_{t}\right)$. Equation (15) looks familiar from equilibrium statistical mechanics if one replaces $\mathcal{X}_{S}^{*}\left(\lambda_{t}\right)$ by $\mathcal{X}_{S}\left(\lambda_{t}\right)$, where $\mathcal{X}$ is used to denote $\mathcal{F}, \mathcal{U}$, or $\mathcal{S}$. Furthermore, it follows from $\mathcal{Z}_{S}^{*}\left(\lambda_{t}\right)=$ $\mathcal{Z}_{S B}\left(\lambda_{t}\right) / \mathcal{Z}_{B}$ that a certain additivity property holds:

$$
\mathcal{X}_{S}^{*}\left(\lambda_{t}\right)=\mathcal{X}_{S B}\left(\lambda_{t}\right)-\mathcal{X}_{B}
$$

This implies, e.g., that the equilibrium system internal energy plus the equilibrium internal energy of the bare, unperturbed bath is equal to the global internal energy of the system-bath composite. The energy and entropy of the system, however, remain in general not additive. Indeed, if the system $S$ is split into two subsystems, $S=X \otimes Y$, and if one follows the same logic as above by assigning $\mathcal{X}_{X}^{*}\left(\lambda_{t}\right) \equiv \mathcal{X}_{X Y B}\left(\lambda_{t}\right)-\mathcal{X}_{Y B}$ and $\mathcal{X}_{Y}^{*}\left(\lambda_{t}\right) \equiv \mathcal{X}_{X Y B}\left(\lambda_{t}\right)-\mathcal{X}_{X B}$ to $X$ and $Y$, respectively, it no longer holds true that $\mathcal{X}_{X}^{*}+\mathcal{X}_{Y}^{*}+\mathcal{X}_{B}=\mathcal{X}_{X Y B}$, i.e., $\mathcal{X}_{X}^{*}+$ $\mathcal{X}_{Y}^{*} \neq \mathcal{X}_{X Y}^{*}$ in general.

Another choice arises if one is only interested in the coarsegrained thermodynamics of an extended system, $S^{\prime}=S \otimes R$, which by incorporating part of the bath, $R$, can be treated as weakly coupled to the remaining part of the bath. This strategy, which is based on tools from Refs. [41-43], can 
be used to show that for classical dynamics the following definition emerges naturally [11]:

$$
\tilde{F}_{S}(t) \equiv F_{S}^{\mathrm{CG}}(t)=F_{S}^{*}(t)+\mathcal{F}_{R}
$$

Here, "CG" stands for coarse-graining and $\mathcal{F}_{R}$ is the equilibrium free energy of the part of the bath that was incorporated in the extended system which obviously has no impact on the change in system nonequilibrium free energy as $\Delta F_{S}^{\mathrm{CG}}(t)=$ $\Delta F_{S}^{*}(t)$. Furthermore, relations formally identical to Eqs. (15) and (16), but in each case with a redefined equilibrium value, can be also derived. A crucial observation made in Ref. [11] was that the so-defined thermodynamic quantities $F_{S}^{\mathrm{CG}}(t)$, $U_{S}^{\mathrm{CG}}(t)$, and $S_{S}^{\mathrm{CG}}(t)$ capture the full nonequilibrium thermodynamics of the weakly coupled open system $S^{\prime}=S \otimes R$ in the limit where the remaining degrees of freedom $R$ are fast and can be adiabatically eliminated, i.e., whenever they can be approximated to be in a conditional equilibrium state. Even beyond that limit, so-called Markovian embedding strategies can be used to study the thermodynamics of strongly coupled open quantum systems [7,44-50].

We remark that all the results mentioned so far are powerful because they are exact mathematical identities that hold for any arbitrary system-bath Hamiltonian dynamics, and in particular any bath size.

\section{LOCAL MEASURABILITY OF THE HAMILTONIAN OF MEAN FORCE}

In the previous section we have reviewed a thermodynamic framework, where all thermodynamic quantities can be evaluated based solely on knowledge of the reduced system state $\rho_{S}(t)$. From the point of open quantum system theory [28] this makes it an appealing theoretical framework. Also experimentally, while still challenging, quantum state tomography of $\rho_{S}(t)$ has been already demonstrated for many technologically relevant platforms. Classically, one can directly use stochastic trajectories to evaluate the corresponding stochastic thermodynamic quantities.

However, there is one caveat: evaluating many thermodynamic quantities, such as the free energy (5), requires knowledge of the HMF (2). In particular, the partition function $\mathcal{Z}_{S}^{*}\left(\lambda_{t}\right)=\mathcal{Z}_{S B}\left(\lambda_{t}\right) / \mathcal{Z}_{B}$ cannot be inferred from the reduced system state (1) alone. Instead, it is fixed by the ratio of partition functions of the system-bath composite and the bath alone. This is not only theoretically challenging to compute, but it also seems experimentally out of reach.

We here overcome this severe practical limitation in the following sense. First, we show that there is an amount of freedom involved in defining the HMF, meaning that the partition function $\mathcal{Z}_{S}^{*}\left(\lambda_{t}\right)$ and therefore the thermostatics will be different but the thermodynamics remains unchanged. Second, we demonstrate that this freedom can be used to construct a strong-coupling thermodynamics based solely on local measurements of the system. Importantly, this is done in a modelindependent way, based only on three minimal assumptions: the ability to measure the system state, knowledge of the system Hamiltonian, and knowledge of the bath temperature.
We start by emphasizing again that the reduced state of $\pi_{S B}\left(\lambda_{t}\right)$,

$$
\pi_{S}^{*}\left(\lambda_{t}\right)=\operatorname{tr}_{B}\left\{\pi_{S B}\left(\lambda_{t}\right)\right\}=\frac{e^{-\beta \tilde{H}_{S}\left(\lambda_{t}\right)}}{\tilde{\mathcal{Z}}_{S}\left(\lambda_{t}\right)},
$$

does not uniquely determine $\tilde{H}_{S}\left(\lambda_{t}\right)$ and $\tilde{\mathcal{Z}}_{S}\left(\lambda_{t}\right)$. Fixing one, however, determines the other. Next, we demonstrate that any choice of $\tilde{\mathcal{Z}}_{S}\left(\lambda_{t}\right)$, which fulfills

$$
\frac{\tilde{\mathcal{Z}}_{S}\left(\lambda_{t}\right)}{\tilde{\mathcal{Z}}_{S}\left(\lambda_{0}\right)}=\frac{\mathcal{Z}_{S}^{*}\left(\lambda_{t}\right)}{\mathcal{Z}_{S}^{*}\left(\lambda_{0}\right)}
$$

does not change the thermodynamics. Equivalently, we can say that any choice that fixes the differences of the HMFs, i.e., $\Delta H_{S}^{*}\left(\lambda_{t}\right)=\Delta \tilde{H}_{S}\left(\lambda_{t}\right)$, does not change the thermodynamics. This can be checked as follows. First, one expresses the original HMF in terms of the effective HMF from Eq. (18) as

$$
H_{S}^{*}\left(\lambda_{t}\right)=\tilde{H}_{S}\left(\lambda_{t}\right)+\frac{1}{\beta} \ln \frac{\tilde{\mathcal{Z}}_{S}\left(\lambda_{t}\right)}{\mathcal{Z}_{S}^{*}\left(\lambda_{t}\right)} .
$$

Notice that the second term on the right-hand side is just a real number and can be taken out of any trace operation. Using this insight, one readily verifies with the help of Eq. (19) that the thermodynamics (i.e., heat, work, change in internal energy and system entropy, entropy production) is insensitive to this redefinition. This is even true for quantities defined at the stochastic level. Therefore, we conclude that all choices fulfilled by Eq. (19) are equally legitimate starting points to construct a theory of nonequilibrium thermodynamics.

Experimentally, reconstructing $\tilde{H}_{S}\left(\lambda_{t}\right)$ can be done in various ways, in particular in the classical case. For instance, assume that we know the reduced system equilibrium states $\pi_{S}^{*}\left(\lambda_{t}\right)$ for all relevant values $\lambda_{t}$ of the control protocol. This state can be inferred by doing only measurements of the system. Then, set

$$
\tilde{H}_{S}\left(\lambda_{t}\right)=-T\left[\ln \pi_{S}^{*}\left(\lambda_{t}\right)+\ln \tilde{\mathcal{Z}}_{S}\left(\lambda_{t}\right)\right]
$$

which still does not fully fix $\tilde{H}_{S}\left(\lambda_{t}\right)$ as we do not know the constant $\tilde{\mathcal{Z}}_{S}\left(\lambda_{t}\right)$. However, now we make use of the freedom mentioned above. For this purpose we fix one of the partition functions, say the one at time $t=0, \tilde{\mathcal{Z}}_{S}\left(\lambda_{0}\right)$, to a known value. This value is completely arbitrary [51] and fixes $\tilde{H}_{S}\left(\lambda_{0}\right)$. To fix $\tilde{H}_{S}\left(\lambda_{t}\right)$ for all other times $t \neq 0$, we choose $\tilde{\mathcal{Z}}_{S}\left(\lambda_{t}\right)$ such that Eq. (19) is fulfilled, which only requires us to infer $\mathcal{Z}_{S}^{*}\left(\lambda_{t}\right) / \mathcal{Z}_{S}^{*}\left(\lambda_{0}\right)$. One way to do so is immediately offered by Eq. (10) after recognizing that $e^{-\beta \Delta \mathcal{F}_{S}^{*}\left(\lambda_{t}\right)}=\mathcal{Z}_{S}^{*}\left(\lambda_{t}\right) / \mathcal{Z}_{S}^{*}\left(\lambda_{0}\right)$. Note that, in the classical case, the left-hand side of Eq. (10) can be evaluated by only knowing the stochastic work, which can be inferred by measuring only system trajectories.

We comment on another possibility to infer $\tilde{H}_{S}\left(\lambda_{t}\right)$ in a classical setting provided that we fixed $\tilde{\mathcal{Z}}_{S}\left(\lambda_{0}\right)$ to an arbitrary value. For this purpose we return to Eq. (7). By using Eqs. (4) and (5), we see that

$$
\Delta\left\langle H_{S}^{*}\left(\lambda_{t}\right)\right\rangle=W(t)+T \Delta S_{\mathrm{Sh}}\left[\rho_{S}(t)\right]-T \Sigma(t) .
$$

Here, $\Delta\left\langle H_{S}^{*}\left(\lambda_{t}\right)\right\rangle=\operatorname{tr}_{S}\left\{H_{S}^{*}\left(\lambda_{t}\right) \rho_{S}(t)-H_{S}^{*}\left(\lambda_{0}\right) \rho_{S}(0)\right\}$ denotes the change in expectation value of the HMF and $\Delta S_{\mathrm{Sh}}\left[\rho_{S}(t)\right]$ denotes the change in Shannon entropy of the classical distribution $\rho_{S}(t)$. Now, notice that the right-hand side of Eq. (22) 
is completely determined by knowing $\rho_{S}(t)$ and $\pi_{S}^{*}\left(\lambda_{t}\right)$, but knowledge of the HMF is not required to evaluate it. Next, we use Eqs. (19) and (20) to deduce that $\Delta\left\langle H_{S}^{*}\left(\lambda_{t}\right)\right\rangle=$ $\Delta\left\langle\tilde{H}_{S}\left(\lambda_{t}\right)\right\rangle$. Hence,

$$
\begin{aligned}
\operatorname{tr}_{S}\left\{\tilde{H}_{S}\left(\lambda_{t}\right) \rho_{S}(t)\right\}= & W(t)+T \Delta S_{\mathrm{Sh}}\left[\rho_{S}(t)\right] \\
& -T \Sigma(t)+\operatorname{tr}_{S}\left\{\tilde{H}_{S}\left(\lambda_{0}\right) \rho_{S}(0)\right\} .
\end{aligned}
$$

Except $\tilde{H}_{S}\left(\lambda_{t}\right)$, all quantities are known in this expression and can be inferred by measuring the system only. To finally reconstruct $\tilde{H}_{S}\left(\lambda_{t}\right)$ from this expression, we need a set of final states $\left\{\rho_{S}(t)\right\}$, which are independent and linearly span the probability space. Such a set can be generated, e.g., by using initial states different from $\pi_{S}^{*}\left(\lambda_{0}\right)$ (as allowed in the classical regime [6]) or by using different driving protocols $\left\{\lambda_{s} \mid 0 \leqslant s \leqslant t\right\}$ keeping $\lambda_{t}$ at time $t$ fixed. In contrast to the previously mentioned approach, Eq. (23) might be particularly convenient from a numerical point of view as it only requires knowledge about the ensemble of states $\rho_{S}(t)$.

We now turn to the quantum case, where the problem is more complicated as the generalization of Eq. (10) can be only derived using the TPPEMS. This is experimentally demanding. To circumvent this problem, we consider an adiabatically slow process in which $\rho_{S}(t)=\pi_{S}^{*}\left(\lambda_{t}\right)$ for all times $t$ and we assume the second law (4) becomes an equality: $W(t)=$ $\Delta \mathcal{F}_{S}^{*}\left(\lambda_{t}\right)$. Note that, in contrast to the previous results, the latter is not an exact identity for a finite-size heat bath. Instead, we here have to assume that the system-bath composite is coupled to a "superbath" of inverse temperature $\beta$. Then, to make the operational meaning of this approach transparent, we express the second law as

$$
\int_{0}^{t} d s \operatorname{tr}_{S}\left\{\frac{\partial H_{S}\left(\lambda_{s}\right)}{\partial s} \pi_{S}^{*}\left(\lambda_{s}\right)\right\}=-T \ln \frac{\mathcal{Z}_{S}^{*}\left(\lambda_{t}\right)}{\mathcal{Z}_{S}^{*}\left(\lambda_{0}\right)} .
$$

This again completely fixes the ratio of partition functions and thus, the HMF up to an irrelevant degree of freedom. Note that, in theory, such an adiabatic process requires infinite time. However, compared to the weak-coupling regime, strong coupling might be helpful here as the relaxation timescales are larger and hence, we can implement the process faster. Furthermore, note that Eq. (24) does not require one to perform any measurement of work per se, but is fully accessible by quantum process tomography.

\section{THE CRITICISM OF TALKNER AND HÄNGGI}

In two recent papers [52,53] Talkner and Hänggi (abbreviated T\&H in the following) critically questioned the approach reviewed in Sec. I. Before turning to their three main points of criticism, we review what $\mathrm{T} \& \mathrm{H}$ take for granted and do not question. In accordance with Refs. [1-20] this includes the assumption that the initial state can be taken to be a global Gibbs state $\pi_{S B}\left(\lambda_{0}\right)$ and that the average work in both the quantum and the classical case, is given by Eq. (3) [54]. They therefore start from the same premise as we did in Sec. I.

Furthermore, T\&H fix the definition of equilibrium energy and entropy by the relations (15) based on the choice $\mathcal{F}_{S}^{*}\left(\lambda_{t}\right)=-T \ln \mathcal{Z}_{S}^{*}\left(\lambda_{t}\right)$. This choice is referred to as "thermodynamically consistent" [53]. But as discussed above, this choice is not unique if one only requires the differences in thermodynamic state functions to be reproduced and not their absolute value. We note that related questions arise on the ongoing discussion of how to correctly account for the interaction energy in simple mesoscopic systems [21-23,5559].

We now briefly summarize the three main points of criticism by $\mathrm{T} \& \mathrm{H}$ :

(a) Since the HMF together with its conventional used normalization [see Eq. (2)] requires precise measurements of $\mathcal{Z}_{S}^{*}\left(\lambda_{t}\right)=\mathcal{Z}_{S B}\left(\lambda_{t}\right) / \mathcal{Z}_{B}$, T\&H "emphasize that the HMF does not follow from the reduced state of the open system" and, without additional knowledge, "the HMF remains undetermined" and finding it for real systems "presents in practice an impossible task" [53].

(b) When trying to construct the corresponding fluctuating thermodynamic potentials along a single trajectory in view of the classical framework of stochastic thermodynamics, there is a vast amount of ambiguity left. Thus, "the stochastic energetics suffers from the problem [of ambiguity]" and "the same flaw also adheres to stochastic thermodynamics" [53]. Furthermore, T\&H write that "other restrictions on the hypothetical fluctuating thermodynamic potentials are not known" [53].

(c) Points (a) and (b) were first put forward in the classical context [52]. In addition, in the quantum case $\mathrm{T} \& \mathrm{H}$ write that "it is not possible to specify [...] simultaneously work and heat, not even their averages" and any "formulation of a first law for other than weakly interacting quantum systems [...] seems doubtful" [53].

Our reply to this criticism is as follows: Concerning point (a), the main technical contribution of the present Rapid Communication directly addresses point (a) since we provide a clear experimental prescription to determine the HMF, up to a thermodynamically irrelevant constant, by local measurements of the system only. This is an important result: although the open system dynamics of $\rho_{S}(t)$ depends strongly on the the details of $V_{S B}$ and $H_{B}$, no knowledge of them is required to experimentally infer the thermodynamics of the open system. Thus, the criticism of T\&H expressed in point (a) remains formally correct-the HMF together with the particular choice (2) of partition function is not measurable using only knowledge about $\rho_{S}(t)$ - but this has no thermodynamic consequences if we choose a partition function obeying Eq. (19). This less restrictive choice of the partition function can be experimentally inferred based only on knowledge about $\rho_{S}(t)$.

Concerning point (b), we first note that the ambiguities in Eqs. (53) and (54) discussed in Ref. [52] are absent if one takes into account that they have to vanish on average for all possible time-evolved nonequilibrium states as we show in [60]. Second, the fluctuating thermodynamic potentials must satisfy the second law (4) or the classical fluctuation theorem (11), which indeed constitute further restrictions.

Concerning point (c), the correct identification of heat and work in the quantum regime is more subtle as there is still no consensus on these questions. The main objection of T\&H is based on their assessment that heat and work are like "position and momentum," whose values "can not be assigned [simultaneously]," and the measurements "need to be error free" and the "energy value must be detected with certainty" 
[53]. However, such measurements are never strictly realized in any quantum experiment and one way to address this issue is to construct a thermodynamic framework that takes into account incomplete information, as recently proposed in Refs. [18,20,61,62] (see also Ref. [63]). This approach provides consistent definitions of heat and work based on the available information in an experiment and does not assume perfect measurements of the bath like the TPPEMS. It also reduces to previously explored cases in the literature in its respective limit.

\section{CONCLUSION}

The thermodynamic framework based on the HMF provides a solid and, as we have shown, operationally meaningful approach to formulate nonequilibrium thermodynamics in the strong-coupling regime. It nevertheless has its limitations. Most importantly, it does not extend to the experimentally relevant situation of multiple heat baths, where only a few formally exact results are known $[29,30,35]$ and a couple of promising theoretical tools, restricted to particular models, were devised [7,21,23,44-50,56-59,64-75].

To conclude, strong-coupling nonequilibrium thermodynamics is not as straightforward as its weak-coupling counterpart and more care is required when specifying the experimental setup including the different classes of possible system preparations. Yet, we are convinced that this quest brought important progress and will continue to do so.

\section{ACKNOWLEDGMENTS}

P.S. acknowledges fruitful discussions with Ángel Rivas. Correspondence with Peter Hänggi and Peter Talkner is gratefully acknowledged. P.S. is financially supported by the DFG (project STR 1505/2-1) and also acknowledges funding from the Spanish MINECO FIS2016-80681-P (AEI-FEDER, UE). M.E. is supported by the European Research Council project NanoThermo (ERC-2015-CoG Agreement No. 681456).
[1] C. Jarzynski, J. Stat. Mech. (2004) P09005.

[2] M. Campisi, P. Talkner, and P. Hänggi, Phys. Rev. Lett. 102, 210401 (2009).

[3] M. Campisi, P. Talkner, and P. Hänggi, J. Phys. A.: Math Theor. 42, 392002 (2009).

[4] S. Hilt, S. Shabbir, J. Anders, and E. Lutz, Phys. Rev. E 83, 030102(R) (2011).

[5] L. Pucci, M. Esposito, and L. Peliti, J. Stat. Mech. (2013) P04005.

[6] U. Seifert, Phys. Rev. Lett. 116, 020601 (2016).

[7] P. Strasberg, G. Schaller, N. Lambert, and T. Brandes, New. J. Phys. 18, 073007 (2016).

[8] T. G. Philbin and J. Anders, J. Phys. A: Math. Theor. 49, 215303 (2016).

[9] C. Jarzynski, Phys. Rev. X 7, 011008 (2017).

[10] H. J. D. Miller and J. Anders, Phys. Rev. E 95, 062123 (2017).

[11] P. Strasberg and M. Esposito, Phys. Rev. E 95, 062101 (2017).

[12] E. Aurell, Entropy 19, 595 (2017).

[13] M. Perarnau-Llobet, H. Wilming, A. Riera, R. Gallego, and J. Eisert, Phys. Rev. Lett. 120, 120602 (2018).

[14] E. Aurell, Phys. Rev. E 97, 042112 (2018).

[15] J.-T. Hsiang and B. L. Hu, Entropy 20, 423 (2018).

[16] H. J. D. Miller and J. Anders, Nat. Commun. 9, 2203 (2018).

[17] P. Strasberg and M. Esposito, Phys. Rev. E 99, 012120 (2019).

[18] P. Strasberg, Phys. Rev. Lett. 123, 180604 (2019).

[19] T. Herpich, K. Shayanfard, and M. Esposito, Phys. Rev. E 101, 022116 (2020).

[20] P. Strasberg, Quantum 4, 240 (2020).

[21] A. Bruch, M. Thomas, S. Viola Kusminskiy, F. von Oppen, and A. Nitzan, Phys. Rev. B 93, 115318 (2016).

[22] M. A. Ochoa, A. Bruch, and A. Nitzan, Phys. Rev. B 94, 035420 (2016).

[23] P. Haughian, M. Esposito, and T. L. Schmidt, Phys. Rev. B 97, 085435 (2018).

[24] L. Onsager, Chem. Rev. 13, 73 (1933).

[25] J. G. Kirkwood, J. Chem. Phys. 3, 300 (1935).
[26] B. Roux and T. Simonson, Biophys. Chem. 78, 1 (1999).

[27] M. F. Gelin and M. Thoss, Phys. Rev. E 79, 051121 (2009).

[28] H.-P. Breuer and F. Petruccione, The Theory of Open Quantum Systems (Oxford University Press, Oxford, 2002).

[29] D. Andrieux, P. Gaspard, T. Monnai, and S. Tasaki, New J. Phys. 11, 043014 (2009).

[30] M. Esposito, K. Lindenberg, and C. Van den Broeck, New J. Phys. 12, 013013 (2010).

[31] K. Takara, H.-H. Hasegawa, and D. J. Driebe, Phys. Lett. A 375, 88 (2010).

[32] D. Reeb and M. M. Wolf, New J. Phys. 16, 103011 (2014).

[33] K. Ptaszyński and M. Esposito, Phys. Rev. Lett. 123, 200603 (2019).

[34] Á Rivas, Phys. Rev. Lett. 124, 160601 (2020).

[35] P. Strasberg and A. Winter, arXiv:2002.08817.

[36] D. Kondepudi and I. Prigogine, Modern Thermodynamics: From Heat Engines to Dissipative Structures (Wiley, West Sussex, 2007).

[37] M. Esposito, U. Harbola, and S. Mukamel, Rev. Mod. Phys. 81, 1665 (2009).

[38] M. Campisi, P. Hänggi, and P. Talkner, Rev. Mod. Phys. 83, 771 (2011).

[39] See Supplemental Material at http://link.aps.org/supplemental/ 10.1103/PhysRevE.101.050101 for a definition of work and free energy at the stochastic level for classical systems and a proof of Eq. (11).

[40] Quantum mechanically, this calculation is not completely straightforward [15].

[41] U. Seifert, Eur. Phys. J. E 34, 26 (2011).

[42] M. Esposito, Phys. Rev. E 85, 041125 (2012).

[43] S. Bo and A. Celani, J. Stat. Phys. 154, 1325 (2014).

[44] D. Newman, F. Mintert, and A. Nazir, Phys. Rev. E 95, 032139 (2017).

[45] G. Schaller, J. Cerrillo, G. Engelhardt, and P. Strasberg, Phys. Rev. B 97, 195104 (2018).

[46] P. Strasberg, G. Schaller, T. L. Schmidt, and M. Esposito, Phys. Rev. B 97, 205405 (2018). 
[47] S. Restrepo, J. Cerrillo, P. Strasberg, and G. Schaller, New J. Phys. 20, 053063 (2018).

[48] G. Schaller and A. Nazir, Thermodynamics in the Quantum Regime (Springer, New York, 2018), Chap. 23.

[49] S. Restrepo, S. Böhling, J. Cerrillo, and G. Schaller, Phys. Rev. B 100, 035109 (2019).

[50] L. A. Correa, B. Xu, B. Morris, and G. Adesso, J. Chem. Phys. 151, 094107 (2019).

[51] In our opinion a particularly convenient choice is given by the weak-coupling partition function $\tilde{\mathcal{Z}}_{S}\left(\lambda_{0}\right)=\mathcal{Z}_{S}\left(\lambda_{0}\right)=\operatorname{tr}_{S}\left\{e^{-\beta H_{S}\left(\lambda_{0}\right)}\right\}$. Via Eq. (19) this implies $\tilde{\mathcal{Z}}_{S}\left(\lambda_{t}\right)=\mathcal{Z}_{S}^{*}\left(\lambda_{t}\right) \mathcal{Z}_{S}\left(\lambda_{0}\right) / \mathcal{Z}_{S}^{*}\left(\lambda_{0}\right)$, which guarantees that $\tilde{\mathcal{Z}}_{S}\left(\lambda_{t}\right)$ always reduces to $\mathcal{Z}_{S}\left(\lambda_{t}\right)=\operatorname{tr}_{S}\left\{e^{-\beta H_{S}\left(\lambda_{t}\right)}\right\}$ in the weak-coupling limit.

[52] P. Talkner and P. Hänggi, Phys. Rev. E 94, 022143 (2016).

[53] P. Talkner and P. Hänggi, arXiv:1911.11660v2.

[54] In the quantum case, the very meaning of an "average" is $a$ priori not clear as any measurement strategy can disturb the system and change the dynamics. However, if the TPPEMS provides a legitimate way to measure work, as assumed by T\&H [53], then Eq. (3) follows from an ordinary ensemble average over the TPPEMS statistics.

[55] M. F. Ludovico, J. S. Lim, M. Moskalets, L. Arrachea, and D. Sánchez, Phys. Rev. B 89, 161306(R) (2014).

[56] M. Esposito, M. A. Ochoa, and M. Galperin, Phys. Rev. B 92, 235440 (2015).

[57] M. F. Ludovico, M. Moskalets, D. Sánchez, and L. Arrachea, Phys. Rev. B 94, 035436 (2016).

[58] M. F. Ludovico, L. Arrachea, M. Moskalets, and D. Sánchez, Phys. Rev. B 97, 041416(R) (2018).

[59] W. Dou, M. A. Ochoa, A. Nitzan, and J. E. Subotnik, Phys. Rev. B 98, 134306 (2018).

[60] According to Ref. [52], any function $h(\mathbf{x})$ of the system phase space coordinate $\mathbf{x}$ can be added to the fluctuating potentials provided that its average vanishes, $\int d \mathbf{x} h(\mathbf{x}) p(\mathbf{x} ; \beta)=0$. Note that $\mathrm{T} \& \mathrm{H}$ denote with $p(\mathbf{x} ; \beta)$ the (in general) nonequilibrium system distribution at time $t$ (indicating with the parameter $\beta$ that the bath is initially in a conditional equilibrium state with respect to that inverse temperature). The system, however, can be prepared and driven arbitrarily, which implies that $\int d \mathbf{x} h(\mathbf{x}) p(\mathbf{x} ; \beta)=0$ must hold for all possible time-evolved system states $p(\mathbf{x} ; \beta)$. As those states typically form a complete set of independent basis states, it follows that $h(\mathbf{x})=0$ for each time $t$.

[61] P. Strasberg, Phys. Rev. E 100, 022127 (2019).

[62] P. Strasberg and A. Winter, Phys. Rev. E 100, 022135 (2019).

[63] A. E. Allahverdyan and T. M. Nieuwenhuizen, Phys. Rev. E 71, 066102 (2005).

[64] G. Schaller, T. Krause, T. Brandes, and M. Esposito, New J. Phys. 15, 033032 (2013).

[65] M. Esposito, M. A. Ochoa, and M. Galperin, Phys. Rev. Lett. 114, 080602 (2015).

[66] D. Gelbwaser-Klimovsky and A. Aspuru-Guzik, J. Phys. Chem. Lett. 6, 3477 (2015).

[67] C. Wang, J. Ren, and J. Cao, Sci. Rep. 5, 11787 (2015).

[68] M. F. Ludovico, L. Arrachea, M. Moskalets, and D. Sánchez, Entropy 18, 419 (2016).

[69] A. Kato and Y. Tanimura, J. Chem. Phys. 145, 224105 (2016).

[70] G. Katz and R. Kosloff, Entropy 18, 186 (2016).

[71] A. Mu, B. K. Agarwalla, G. Schaller, and D. Segal, New J. Phys. 19, 123034 (2017).

[72] A. Bruch, C. Lewenkopf, and F. von Oppen, Phys. Rev. Lett. 120, 107701 (2018).

[73] R. S. Whitney, Phys. Rev. B 98, 085415 (2018).

[74] G. Guarnieri, G. T. Landi, S. R. Clark, and J. Goold, Phys. Rev. Res. 1, 033021 (2019).

[75] C. Hsieh, J. Liu, C. Duan, and J. Cao, J. Phys. Chem. C 123, 17196 (2019). 